\title{
Regenerative Medicine: Transforming the Drug Discovery and Development Paradigm
}

\author{
Sotirios K. Karathanasis \\ Cardiovascular \& Metabolic Diseases, Gaithersburg, Maryland 20878 \\ Correspondence: karathanasiss@medimmune.com
}

Despite the explosion of knowledge in basic biological processes controlling tissue regeneration and the growing interest in repairing/replacing diseased tissues and organs through various approaches (e.g., small and large molecule therapeutics, stem cell injection, tissue engineering), the pharmaceutical industry (pharma) has been reluctant to fully adopt these technologies into the traditional drug discovery and research and development (R\&D) process. In this article, I discuss knowledge-base gaps and other possible factors that may delay full incorporation of these innovations in pharma R\&D. I hope that this discussion will illuminate key issues that currently limit synergistic relationships between pharma and academic institutions and may even stimulate initiation of such collaborative research.

$\mathrm{B}^{\mathrm{r}}$ reakthroughs in stem cell biology and the clinical evaluation of regenerative therapeutics offer an unprecedented opportunity to transform traditional pharma research and development $(\mathrm{R} \& \mathrm{D})$ and revolutionize future medical practice. Capitalizing on these opportunities will require a significant transformation in the pharma R\&D model, including a shift in the way pharma and academic institutions interact.

Although scientific breakthroughs are driven mostly by academic institutions, conversion of novel biologic insights into successful medical products is dependent on the drug development and distribution infrastructure available in traditional pharma. In this context, successful, purpose-driven innovation at the academia-pharma interface necessitates mutual understanding of the several factors including: general philosophy, long-term vision, opera- tional and cultural paradigms, and key drivers and stressors across both of these enterprises.

To describe how emerging technologies in regenerative medicine could transform pharma $\mathrm{R} \& \mathrm{D}$ and revitalize this entire business sector, I will first outline the current pharma R\&D business environment and the manner in which this environment is creating the need for change in the prevailing business model.

It has been argued that the current pharmaceutical business model is not sustainable because of the productivity crisis in pharma R\&D (Pammolli et al. 2011). Many factors are responsible including escalating $R \& D$ costs, increased pressures for improved performance drugs, increased payer pressures, excessive regulatory stringency, increased focus on high-risk research involving complex therapeutic targets, and an over-reliance on "molecular reduction-

Editors: Margaret Buckingham, Christine L. Mummery, and Kenneth R. Chien

Additional Perspectives on The Biology of Heart Disease available at www.perspectivesinmedicine.org

Copyright (C) 2014 Cold Spring Harbor Laboratory Press; all rights reserved; doi: 10.1101/cshperspect.a014084

Cite this article as Cold Spring Harb Perspect Med 2014;4:a014084 
ism" to deliver solutions to complex biological problems (Scannell et al. 2012). In addition, limitations in human capital with a robust knowledge base in both translational medicine and therapeutics further intensifies the severity of the current predicament (FitzGerald 2010).

Initiatives to "industrialize" and "streamline" the discovery process (Bhattacharya et al. 2005) and improve process efficiency (e.g., the "lean-six-sigma" methodology) (Sewing et al. 2008), are based on last century's Frederick Taylor's "scientific management" paradigm (Taylor 1911) and have been largely unsuccessful in improving $R \& D$ effectiveness. In fact, they may have even contributed to an inward-focused culture lacking sufficient flexibility to internalize novel therapeutic concepts emerging from academia. Indeed, the rate of advance in stem cell biology, regenerative medicine, and nanosciences dramatically outpaces the rate of their incorporation into pharma R\&D.

Taken together, these factors have fueled knowledge-base and innovation deficits in pharma, which will continue to grow unless systemic change is made (Drews and Ryser 1996; U.S. Department of Health and Human Services, Food and Drug Administration 2004; Pammolli et al. 2011).

It is within this contextual landscape that recent breakthroughs in developmental biology, stem cell biology, and regenerative medicine are emerging as disruptive technologies with the potential to revolutionize R\&D and radically transform future medical practice.

\section{PHARMA'S INTEREST IN STEM CELL BIOLOGY AND REGENERATIVE MEDICINE}

Although pharma's initial reaction to the realm of regenerative medicine has been cautious, it is generally recognized that these technologies represent an opportunity for substantial market expansion. Indeed, because of the higher burden of chronic disease driven by the aging world population, healthcare costs are expected to increase dramatically over the next 20 years. It is estimated that by 2030 the elderly population in the United States alone will increase by nearly 32 million (Werner et al. 2011). This will sub- stantially increase the need for more cost-effective therapies for degenerative conditions common in the elderly, such as heart disease, cancer, stroke, pulmonary disease, and diabetes, all of which are amenable to regenerative medicine approaches.

These future demographic trends, medical needs, and financial realities are very visible in pharma's strategic planning horizon and they are a major driver in pharma's emerging interest in stem cell and regenerative technologies. In this context, pharma's interests can be broadly placed in two categories: (1) drug-screening tools, and (2) regenerative therapies. It should also be noted that there is a growing interest in applying these technologies, particularly patient-derived induced pluripotent stem cells (iPSCs), to risk stratify individual patients, ultimately enabling personalized health care, another clearly articulated ambition of pharma.

\section{DRUG-SCREENING TOOLS}

Drug screening involves a series of decisionmaking steps designed to filter out irrelevant compounds and focus on a few promising compounds with therapeutic potential. The core elements of these drug-screening programs typically include three sequential compound-filtering steps: in vitro screening, in-cells screening, and in-animal screening. Compounds meeting prespecified criteria in the in vitro screening assays are progressed into the cell-screening assays, and, if they meet the cell-screening assay, prespecified criteria are further progressed into animal models. Compounds emerging from this sequence of events are further studied in animals to determine their pharmacokinetic and pharmacodynamic (PKPD) properties and dose-dependent separation of molecular target engagement from adverse effects because of nontarget activity. The so-called margins of safety (MoS) must be defined before lead compounds are tested in humans.

Although there is logic to this drug-screening paradigm, the persistent and growing attrition of experimental therapies at all stages of clinical testing, including postapproval, point to inherent flaws regarding translatability to hu- 
mans (Kola and Landis 2004). By necessity, most screening cascades employ nonhuman species, raising the concern that animal disease models currently in use do not recapitulate human disease and, therefore, are of limited translational predictive value.

Similar concerns also apply to the various cell lines used for "compound-filtering." Although engineered cell lines typically used in the early stages of drug-screening cascades help select compounds that trigger pathways mechanistically linked to molecular targets, in general, these cells provide very little information on drug-induced disease-related physiological changes. Efforts to use human-derived cell lines are also plagued with inherent difficulties as cell line behavior may deviate significantly from the parental phenotype, and primary cells have limited expansion potential, undergoing phenotypic drift under prolonged culturing.

The pressing need for reliable models of human disease has fueled a growing interest in pharma to leverage recent developments in stem cell technologies, including stem cell-generated tissues, organs, and chimeric animals, to improve predictivity of drug-screening cascades. The critical importance in this area is also emphasized by the recent initiative by National Institutes of Health (NIH), which provides funding opportunities for research in this area (NIH 2012).

\section{Human Disease-Relevant Cell-Based Models}

Human embryonic stem cells (hESCs) were first isolated 14 years ago (Thomson et al. 1998) and were subsequently differentiated to diverse human cell types (e.g., cardiomyocytes, hepatocytes, and neurons). Pharma has exhibited interest in using hESCs in drug-screening cascades; however, practical implementation has been severely curtailed by a number of problems, including ethical issues of hESCs, limited scalability of hESC culture in vitro, and the lack of hESCs derived from patients with diseases of interest (Grskovic et al. 2011). Yamanaka and coworkers recently reported a method by which an adult terminally differentiated cell can be transformed in vitro to a phenotype similar to
hESCs. This method involves transient expression of a cocktail of transcription factors in patient-derived cells. The resultant cells, iPSCs, can subsequently be differentiated into various cell types, thus facilitating study of medical conditions present in the initial patient (Takahashi and Yamanaka 2006). This technique eliminated ethical concerns associated with ESCs and provided a renewable source of patient-specific pluripotent cells, rejuvenating pharma's interest in the development of cellular models of human disease. This interest has been further fueled by a continuously increasing number of diseasespecific iPSC lines, produced primarily within academic institutions (Grskovic et al. 2011; Robinton and Daley 2012).

Consistent generation and establishment of disease- and patient-specific human cell systems with well-defined phenotypes linked to key aspects of human disease has captured the imagination of both academic and pharma scientists. Clearly, incorporation of such models in drug-screening cascades will improve human translatability and open the possibility of drug "tailoring” for disease subsegments with similar underlying pathoetiologies, ultimately contributing to the other aspiration of pharma: personalized medicine.

Furthermore, the availability of cell systems that closely recapitulate human disease pathoetiology has the potential to usher in a new era of phenotypic screening, whereby compounds are evaluated for their effects on cellular phenotypes rather than prespecified molecular targets, thus addressing the issue of molecular reductionism discussed above. The concept of phenotypic screening is not new to pharma (Swinney and Anthony 2011), but, until recently, cell systems that faithfully recapitulated human disease were not available and previous efforts in this area using alternative systems were largely unsuccessful.

Finally, there are even ambitions to develop "in vitro clinical trials" and "patients in a dish," whereby patient-derived differentiated iPSCs are tested for drug response before the initiation of formal clinical studies. Accurate identification of responders before the initiation of clinical trials could have a marked impact on 
sample size and could provide information about drug effects in off-target tissues (Dimos et al. 2011; Grskovic et al. 2011).

Although in theory iPSCs can give rise to all cell types in an adult organism, consistent differentiation of these cells into mature cell types remains a technical challenge. Commitment and differentiation of pluripotent stem cells into specific lineages is determined by the exact concentration and timing of the necessary paracrine factors and related signaling pathways. In principle, successful differentiation protocols attempt to recapitulate normal human embryonic development, a still-evolving area of research. Nevertheless, in vitro differentiation protocols have been developed for a few cell types such as cardiomyocytes, neurons, and hepatocytes (Dimos et al. 2011; Grskovic et al. 2011).

A notable recent accomplishment in this area is the consistent generation of human white and brown adipocytes from iPSCs and the recapitulation of a human disease-relevant phenotype (insulin resistance) after exposure of these cells to free fatty acids (FFAs), known to be key disease drivers in humans (Ahfeldt et al. 2012).

In addition to the difficulties of consistent differentiation of iPSCs into tissue-specific cell types, there is the issue of differentiated cell maturity. Frequently, differentiated iPSCs exhibit an immature fetal phenotype in vitro, only assuming a mature adult phenotype after grafting in animals (Dimos et al. 2011). Clearly, understanding how the state of maturity of these cells affects the relationship between cell phenotype and disease relevance needs to be further defined before the introduction of the cells into drug-screening cascades.

Finally, the issue of epigenetic somatic memory of iPSCs needs to be addressed. Recent data suggest that epigenetic controls present in the parental cells are preserved in the derived iPSCs and influence their differentiation capacity in favor of lineages closely related to the parental cell (Robinton and Daley 2012). Although such limitations can be overcome by treating with DNA demethylating agents or exposure to specific cytokines, it is important that these issues are addressed up front to avoid po- tential effects of epigenetic factors from irrelevant tissues influencing the phenotype in the ultimate disease model. It should be also noted that any epigenetic contributions to disease expression in disease-relevant tissues are likely to be lost in iPSCs derived from tissues and cells not directly involved in the disease process. However, this could be overcome if iPSCs are derived from tissues either participating in or affected by the disease.

Clearly, robust cell phenotypes closely linked to human disease are essential before the incorporation of iPSC-derived disease models into screening cascades. Although this has been relatively straightforward for monogenic cell autonomous diseases, such as in the case of certain cardiac arrhythmogenic diseases (Grskovic et al. 2011; Oh et al. 2012), it is more challenging with complex multifactorial diseases. For example, diseases such as type 2 diabetes mellitus or atherosclerosis are multifactorial, multiorgan disorders involving a variety of tissues and cell types. In these cases, the relative contribution of each specific cell type to disease expression and a linked disease-relevant cell phenotype needs to be clearly defined before the adoption into drug-screening cascades.

It is possible to induce disease-relevant phenotypes by exposing cells to pathoetiologic factors such as exposure of adipocytes to FFAs to induce adipose tissue insulin resistance (Ahfeldt et al. 2012). Differentiation of iPSCs from a single affected patient into multiple tissue types followed by exposure of the differentiated cells to the same causal stimulus may clarify the relative contribution of different tissue types to a disease state. One such example would include comparison of myocyte and adipocyte response to FFAs in culture to compare their relative contribution to systemic insulin resistance. Glucose-stimulated insulin secretion (GSIS) of pancreatic $\beta$ cells produced from the same patient, in the presence and absence of similar disease-inducing factors, would provide information on the contribution of $\beta$-cell dysfunction. Undoubtedly, the data produced by this type of assay could have major implications for patient-specific drug targeting and personalized healthcare. 
This concept could be expanded further by leveraging the existing knowledge base in the literature on physiological and pathophysiological stimuli known to alter cell behavior in multiple disease states. For example, to study kidney disease, patient-derived podocytes could be treated with glucose or suPAR to induce phenotypic changes in podocyte foot morphology, thought to be a surrogate of the disease phenotype known as podocyte effacement (Wei et al. 2011). Such an assay would not only facilitate translation of drug candidates, but would also enable phenotypic screening to discover novel drugs for kidney diseases. Similarly, exposure of iPSC-derived intestinal L cells (from multiple patients) to drugs designed to increase GLP-1 secretion could delineate patient populations responsive to specific candidate drugs and could also facilitate phenotypic screening for novel drugs capable of inducing intestinal GLP-1 release. This assay could also be used as the starting point for the study of genetic markers associated with drug responsiveness. Furthermore, and particularly relevant to the focus of this collection, a recently reported phenotypic screen for compounds that promote cardiogenesis of ESCs led to discovery of novel compounds that block TGF- $\beta$ signaling by inducing TGF- $\beta$ R2 degradation, thereby selectively directing uncommitted mesodermal cells toward cardiac lineages (Willems et al. 2012). Because of the importance of TGF- $\beta$ signaling in endothelial to mesenchymal transitions (EndMT) and the role of this process in postmyocardial infarction (MI) cardiac fibrosis (von Gise and $\mathrm{Pu} 2012$ ), it will be very exciting to test these compounds for their effects in in vivo MI models. There are many more such examples including patient-derived macrophages for reverse cholesterol transport (RCT) and endothelial cell activation and cholesterol uptake assays for vascular therapies to name only a few (Adams and García-Cardeña 2012).

Another exciting development is the incorporation of human iPSC-derived tissues and organs in whole animals, creating human-animal chimeras. For example, a mouse chimeric model with human iPSC-derived human adipose tissue (Ahfeldt et al. 2012) and a similar model with hESC-derived pancreatic progenitor cells (Schulz et al. 2012) were recently reported. Although such models offer an unprecedented opportunity to study human tissue function in the context of whole organism physiology, longterm preservation of human tissues in such models is dependent on continuous suppression of the recipient animal's immune system. Use of immunosuppressed mouse strains can facilitate this type of experiment, but new solutions may be required to make the chimeric system more widely applicable.

\section{Drug Toxicology and Safety Assays}

Testing candidate drug toxicity in animals before human clinical trials is unreliable. It is often found that compounds that appear safe in animals are toxic in humans. Predictive toxicology using human iPSC-derived tissue cells could enable attrition of compounds with unacceptable safety profiles early in the evaluation process and will also decrease animal use (Wobus and Löser 2011). This is a rapidly advancing area with the European Union 7th Framework Programme "Innovative Medicine Initiative" supporting further research (Innovative Medicine Initiative 2011), and several cell-based predictive toxicity assays are now commercially available. Remaining challenges in this area include scalability of cell culture and production of homogeneous and mature cell populations (Wobus and Löser 2011).

In summary, although there has been remarkable progress over the last few years in establishing human iPSC-derived models of disease, several remaining gaps, such as consistent generation of fully differentiated cells, establishment and validation of disease-relevant phenotypic read-outs, and development of diseaserelevant chimeric human-animal constructs, need be addressed before such technologies are fully integrated within pharma R\&D. Development of human multicellular models that can replicate aspects of human organ physiology, disease pathogenesis, cell-type diversity, and genomic complexity, as envisioned by the NHI RFA mentioned above, has the potential to revolutionize future drug discovery screening. 
S.K. Karathanasis

\section{REGENERATIVE THERAPIES}

The concept of tissue regeneration has been part of human civilization for millennia. Indeed, the ancient Greek legend of Prometheus includes mention of liver regeneration. According to the legend, Prometheus, a deity who took pity on mortals, stole fire from Zeus to bring them light and comfort. Zeus, angered by this gesture, punished Prometheus by tethering him to a rock and sending an eagle to eat his liver each day. Overnight, the liver would regrow for the eagle's return. Regenerative medicine in humans, in the form of organ transplantation and hematopoietic stem cell transplantation has been performed since the 1950s (Maienschein 2011). More recently, with the re-emergent interest in regenerative therapeutics, there has been a flurry of preclinical and clinical activity in this space. In general, these efforts involve approaches such as cell transplantation or paracrine factor-induced activation of endogenous stem cells using small or large molecules and bioactive materials (biopolymers, nanofibers). In the following paragraphs, I discuss opportunities and gaps that need to be filled before pharma is ready to engage further.

\section{Mesenchymal Stem Cell Autologous and Allogeneic Cell Therapies}

Transplantation of bone marrow (BM)-derived hematopoietic stem cells is now a standard clinical procedure for the treatment of certain hematological conditions. Mesenchymal stem cells (MSCs), a fibroblast-like subpopulation found in $\mathrm{BM}$ and other tissues, have been shown to differentiate into a variety of adult tissues and, after systemic administration, have been shown to be safe regarding tumorigenesis and other adverse side effects (Jung et al. 2012). Indeed, MSC regenerative therapies are being currently tested in a variety of diseases including immune modulation, bone and cartilage repair, cardiac regeneration, and wound repair (Sensebé et al. 2010; Culme-Seymour et al. 2012). It is also noteworthy that such adult tissue-derived stem cell regenerative cell therapies are advancing rapidly in veterinary medicine where there is less regulatory stringency. For example, adult stem cell therapies for equine articular cartilage restoration and chondrogenesis have been shown to improve clinical symptoms (Borjesson and Peroni 2011).

Despite these developments and the increased enthusiasm for such approaches to tissue regeneration, there are clear scientific, clinical, regulatory, and financial risks (McKernan et al. 2010; Tozer 2010; Jung et al. 2012). Scientific and clinical risks include choice of cell donor. Although autologous cells would be preferable, donor age appears to affect the efficacy of the isolated cells. It has been shown that aging significantly reduces survival and differentiation potential of BM-MSC (Roobrouck et al. 2008). This age-dependent regenerative decline of endogenous stem cells, referred to as "stem cell aging," is thought to be driven by several factors in the tissue niche from which the adult cells are derived, including inflammation and excessive oxidative stress present in the disease context. These age-related changes in stem cell phenotype and behavior may significantly impact the regenerative potential of cell-replacement therapies for tissue regeneration (Jasper and Kennedy 2012).

Reliable scale production of the desired phenotype is necessary, but not sufficient, for a successful cell-based therapeutic strategy. Issues pertaining to delivery of the therapeutic cell to the target organ would need to be solved. Reported attempts to deliver putative therapeutic cells to the heart exemplify the challenges associated with cell delivery (Ptaszek et al. 2012). Even if a cell can be delivered to the target organ in a safe manner, which ensures optimal retention at the site, survival and maturation of this cell in situ are not guaranteed (Le Huu et al. 2012).

It is also worth noting that the discovery of iPSCs generated a rush of enthusiasm about using patient-derived iPS cells for cell transplantation. However, a number of concerns such as risk for tumorgenicity, epigenetic memory, and differentiated cell purity need to be addressed before this vision becomes reality (Vitale et al. 2011). 
The mechanism by which transplanted cells influence cardiac physiology is strongly debated, with a number of leading investigators in the field arguing that most of the beneficial effects are a result of paracrine effects rather than direct participation of the transplanted cells in tissue regeneration (Penn et al. 2011). Some investigators further argue that stem cells are not even necessary as secreted factors may be entirely responsible for the benefits observed clinically. This, together with the need to improve the endogenous microenvironment, suggests that alternative approaches involving delivery of paracrine and other trophic factors may hold promise.

From the regulatory perspective, relevant issues include: (1) cell characterization and quality control (identity, purity, potency, tumorgenicity, chromosomal stability, and process validation), (2) biodistribution (ability to track cells into different microenvironments and tissues), (3) disease relevance of animal models (large animal models reflecting the disease indication are preferable for surgical implantation and follow-up of cell products), (4) in vivo differentiation (desired mode of action), (5) immune rejection and persistence, (6) clinical pharmacodynamic biomarkers (to follow the differentiation status of the cells during treatment), (7) clinical pharmacokinetic markers (to follow the administered cells during the study), (8) clinical dose finding, (9) efficacy on clinically meaningful endpoints/overall efficiency, and (10) safety regarding teratomas and pathological self-renewal (Halme and Kessler 2006; European Medicines Agency 2011).

From the business perspective, relevant issues include: (1) manufacturing (regulations covering product manufacturing, packaging, facility validation, consistency and safety of materials, sterility of the product, and, where appropriate, sterilization and viral inactivation procedures), (2) logistics (living cell products require precisely controlled conditions and may require cryopreservation and implementation of cold chain logistics and distribution capabilities), (3) reimbursement, and (4) sales and marketing (awareness and understanding of consumers and health care providers of regenerative medicines therapies) (Tozer 2010).
In summary, although a recently published comprehensive review on ongoing cell therapy trials ends with the optimistic note that "overall, these data are highly encouraging for the emerging cell therapy industry, given the early stage of the technology platform in its life cycle, total number of trials, the spread of clinical trial phases and the range of medical indications" (Culme-Seymour et al. 2012), there remains significant challenges before this becomes a routine approach to discovering and developing new medicines.

\section{Paracrine Factors and Developmental Pathway Modulators}

Use of paracrine factors as cardiac regenerative therapeutics is based on the premise that the heart has intrinsic regenerative capacity and that progenitor cells in the adult heart, if adequately stimulated, could influence the heart's response to injury. It is now largely accepted that the beneficial effects observed in trials associated with putative cardiac stem cells are the result of paracrine factor release from these cells, rather than residence of delivered cells in the heart (Penn et al. 2011).

Although amphibians and certain fish are known to maintain a robust ability for cardiac regeneration throughout life, until recently, this was thought to be absent in mammals. In fact, many of the earliest cardiac regenerative therapies were designed based on the assumption that cardiac cells possessed no regenerative capacity. However, recent work has shown that cardiac regenerative activity does exist in mice, but extinguishes very rapidly after birth (Porrello et al. 2011). An evolving body of work using mouse models has revealed the presence of a progenitor population that persists on the epicardial surface of the adult heart. These cells, referred to as epicardial progenitor-derived cells (EPDCs), are stimulated to divide after cardiac injury (e.g., myocardial infarction) but are not independently capable of reversing injury. This cell population is, therefore, a compelling target for paracrine factor-based therapeutics (Smart et al. 2012).

The growing understanding of the networks of signaling events that control early cardiac 
progenitor cell specification and differentiation, such as the sequential activation of the Notch, Wnt/ $\beta$-catenin, and BMP (Klaus et al. 2012), and the knowledge that some of these systems regulate stem cell proliferation and differentiation in the adult heart, raise the possibility that small molecule modulators of these signaling systems could redirect stem cell fate in the diseased adult heart leading to clinically effective cardiac regeneration. For example, it was recently shown that canonical Wnt signaling limits cell renewal of an adult stem cell population known as cardiac side population (CSP), and blocks their ability to contribute to endogenous cardiac regeneration. These data suggest that Wnt signaling inhibitors exert potent cardioprotective effects after myocardial injury (Oikonomopoulos et al. 2011).

Inspired by such a possibility, several investigators have employed phenotypic screens to identify molecules with cardiac regeneration potential. A recent screen in zebrafish using heart size as a screening end point yielded certain Wnt inhibitors (cardionogens) shown to induce cardiogenesis during and after gastrulation (Ni et al. 2011). Similarly, a mouse ESC phenotypic screen identified a 1,4-dihydropyridine selective TGF- $\beta$ inhibitor that promotes cardiogenesis and beating cell clusters in mESC when added between days three and five of differentiation (Willems et al. 2012).

Furthermore, modulation of the paracrine factor milieu via delivery of specific growth factors and chemokines to the myocardial tissue has been reported to lead to effective cardiac regeneration. Cardiac regeneration has been observed with TB4 (Smart et al. 2011), VEGF (Lin et al. 2012), SCF (Yaniz-Galende et al. 2012), and SDF-1, which has recently shown promise in improving heart function in patients with severe heart failure (Penn et al. 2012).

It should be also mentioned that an alternative approach to cardiac regeneration involving direct reprogramming of endogenous fibroblasts into functional cardiomyocytes has also been recently reported. Although this offers the exciting possibility to regenerate the myocardium, reduce scar size, and improve cardiac function, these benefits need to be confirmed in large animal models with thicker myocardium similar to that of humans (Bruneau 2012).

Although this paracrine therapy approach is conceptually appealing and consistent with the current pharma business model for molecule optimization and delivery to the marketplace, there are questions that need to be addressed.

For example, if the replicative and chronological aging as well as the scar tissue and other pathogenic factors affect the functional capacity and regenerative potential of endogenous stem cells in heart disease patients, as has been suggested, the therapeutic effectiveness of paracrine therapies could be hampered (Mohsin et al. 2011). Encouragingly, however, a recent comparison of the postinfarction myogenic potential of neonatal and adult hearts revealed that the failure of adult postinfarct myogenesis is not because of context-dependent restriction of precursor differentiation, but rather because of the distinct developmental potential of adult and neonatal heart progenitor cells (Jesty et al. 2012). Elucidation of key mechanisms and discovery of critical molecular cues that control stem cell development may reveal approaches for resetting the developmental potential of stem cells in adult heart disease patients, thereby allowing recapitulation of neonatal heart regeneration and improved cardiovascular function.

Avery recent example along these lines is the cardiac regeneration and improved heart function observed after adenoviral gene transfer of the c-kit ligand stem cell factor (SCF) into the infarcted myocardium. The experimental evidence suggests that transient overexpression of SCF in the heart increases recruitment of c-kit ${ }^{+}$ cardiac cells and leads to regeneration via activation of the Wnt signaling (Yaniz-Galende et al. 2012). Similarly, elucidation of mechanochemical pathways responsible for transmitting tissue rigidity signals that control cardiac stem cell proliferation and cell-fate choices may allow chemical reprogramming of the patient cardiac mechanical environment in line with the regenerative strategy therapeutic objectives (Kshitiz et al. 2012).

It should also be noted that, although up to now only individual paracrine factors have been explored, robust and effective cardiac regenera- 
tion may require a combination of paracrine factors. Therefore, a challenge for the future is to define such paracrine factor cocktails and tailor them toward indication-specific cardiac pathobiologies as the inherent paracrine factor environment may differ significantly between acute myocardial infarction and chronic heart failure (Penn et al. 2011).

It should also be emphasized that the overall strategy to reengage powerful developmental systems and direct them toward new tissue genesis and organ re-engineering is associated with obvious safety risks. To ameliorate these concerns, there is a need for effective local delivery systems. This is an important area of research and progress is regularly reported in the literature. Recently, self-assembling peptide nanofibers were used for delivery of VEGF (Lin et al. 2012) and collagen-chitosan hydrogels were used for delivery of TB4 (Chiu et al. 2012) both resulting in enhanced cardiac regeneration.

In conclusion, this is a very exciting area of research with the potential to deliver novel medicines that will radically change current medical practice for the treatment of heart diseases. In addition, it should be reiterated that these paracrine and developmental pathway modulator approaches fit perfectly within the current pharma $R \& D$ paradigm providing a unique incentive for pharma to contribute their strong drug $\mathrm{R} \& \mathrm{D}$ experience to focused pharma/academia collaborations aiming to explore novel therapeutic concepts and deliver efficient clinical proof of concepts.

\section{CONCLUDING REMARKS}

Pharma is in urgent need to turn around and revitalize their business model. Innovative technologies such those offered by stem cell biology and regenerative medicine promise a disruptive, "step-change" transformation of the current pharma R\&D model and the opportunity to deliver medicines that will radically alter future medical practice (Denoon and Vollebregt 2010). Although sensationalization of the medical potential of regenerative medicine is unavoidable and has raised concerns about inflated expecta- tions (Tozer 2010), the focused research activities in this area by academia, government (including the NHI and IMI initiatives), and private enterprises assure timely resolution of remaining gaps and actualization of the immense clinical value of these technologies in the near future. Pharma's primary strengths are the process by which lead compounds are turned into a marketable drug, which is a formidable process that includes large-scale production, distribution, quality control, and interactions with regulatory agencies. Also included are structuring and conducting clinical trials, as well as reimbursement and lobbying, which are essential for this emerging business sector to mature into a sustainable business enterprise (Denoon and Vollebregt 2010). Given the complementary strengths of academic institutions and their skills in identification and validation of novel therapeutic targets, a collaborative approach between pharma and academia is essential to bring the exciting potential of regenerative therapeutics into a reality.

\section{ACKNOWLEDGMENTS}

I am truly indebted to my colleagues Lauren Drowley and Richard Lawson, AstraZeneca, and Leon Ptaszek, MGH, Harvard, for their thoughtful reading of the manuscript and their many suggestions for improvement.

\section{REFERENCES}

Adams WJ, García-Cardeña G. 2012. Novel stem cell-based drug discovery platforms for cardiovascular disease. $J$ Biomol Screen 17: 1117-1127.

Ahfeldt T, Schinzel RT, Lee Y-K, Hendrickson D, Kaplan A, Lum DH, Camahort R, Xia F, Shay J, Rhee EP, et al. 2012. Programming human pluripotent stem cells into white and brown adipocytes. Nat Cell Biol 14: 209-219.

Bhattacharya K, Guttman R, Lyman K, Heath I, Kumaran S, Nandi P, Wu F, Athma P, Freiberg C, Johannsen L, et al. 2005. A model-driven approach to industrializing discovery processes in pharmaceutical research. IBM Systems J 44: $145-162$.

Borjesson DL, Peroni JF. 2011. The regenerative medicine laboratory: Facilitating stem cell therapy for equine disease. Clin Lab Med 31: 109-123.

Bruneau BG. 2012. Direct reprogramming for cardiac regeneration: From dream to reality. Circ Res 110: 1392-1394. 
S.K. Karathanasis

Chiu LL, Reis LA, Momen A, Radisic M. 2012. Controlled release of thymosin $\beta 4$ from injected collagen-chitosan hydrogels promotes angiogenesis and prevents tissue loss after myocardial infarction. Regen Med 7: 523-533.

Culme-Seymour EJ, Davie NL, Brindley DA, Edwards-Parton S, Mason C. 2012. A decade of cell therapy clinical trials (2000-2010). Regen Med 7: 455-462.

Denoon A, Vollebregt E. 2010. Can regenerative medicine save Big Pharma's business model from the patent cliff? Regen Med 5: 687-690.

Dimos JT, Griswold-Prenner I, Grskovic M, Irion S, Johnson C, Vaisberg E. 2011. Induced pluripotent stem cells as human disease models. In Annual reports in medicinal chemistry, Vol. 46 (ed. Macor EJ), pp. 369-383. Academic, San Diego.

Drews J, Ryser S. 1996. Innovation deficit in the pharmaceutical industry. Drug Inf J 30: 97-108.

European Medicines Agency. 2011. Reflection paper on stem cell-based medicinal products. EMEA, Committee for Advanced Therapies (CAT), London.

FitzGerald GA. 2010. Perestroika in pharma: Evolution or revolution in drug development? Mt Sinai J Med 77: $327-332$.

Grskovic M, Javaherian A, Strulovici B, Daley GQ. 2011. Induced pluripotent stem cells-Opportunities for disease modelling and drug discovery. Nat Rev Drug Discov 10: 915-929.

Halme DG, Kessler DA. 2006. FDA regulation of stem-cellbased therapies. N Engl J Med 355: 1730-1735.

Innovative Medicine Initiative. 2011. Human induced pluripotent stem (hiPS) cells for drug discovery and safety assessment (IMI). European Union 7th Framework Programme, Luxembourg.

Jasper H, Kennedy BK. 2012. Niche science: The aging stem cell. Cell Cycle 11: 2959-2960.

Jesty SA, Steffey MA, Lee FK, Breitbach M, Hesse M, Reining S, Lee JC, Doran RM, Nikitin AY, Fleischmann BK, et al. 2012. c-kit ${ }^{+}$precursors support postinfarction myogenesis in the neonatal, but not adult, heart. Proc Natl Acad Sci 109: 13380-13385.

Jung Y, Bauer G, Nolta JA. 2012. Concise review: Induced pluripotent stem cell-derived mesenchymal stem cells: Progress toward safe clinical products. Stem Cells 30: 42-47.

Klaus A, Müller M, Schulz H, Saga Y, Martin JF, Birchmeier W. 2012. Wnt/ $\beta$-catenin and Bmp signals control distinct sets of transcription factors in cardiac progenitor cells. Proc Natl Acad Sci 109: 10921-10926.

Kola I, Landis J. 2004. Can the pharmaceutical industry reduce attrition rates? Nat Rev Drug Discov 3: 711-715.

Kshitiz Hubbi ME, Ahn EH, Downey J, Afzal J, Kim D-H, Rey S, Chang C, Kundu A, Semenza GL, Abraham RM, et al. 2012. Matrix rigidity controls endothelial differentiation and morphogenesis of cardiac precursors. Sci Signal 5: pra41.

Le Huu A, Prakash S, Shum-Tim D. 2012. Cellular cardiomyoplasty: Current state of the field. Regen Med 7: 571582.

Lin Y-D, Luo C-Y, Hu Y-N, Yeh M-L, Hsueh Y-C, Chang MY, Tsai D-C, Wang J-N, Tang M-J, Wei EIH, et al. 2012. Instructive nanofiber scaffolds with VEGF create a mi- croenvironment for arteriogenesis and cardiac repair. Sci Transl Med 4: p146ra109.

Maienschein J. 2011. Regenerative medicine's historical roots in regeneration, transplantation, and translation. Dev Biol 358: 278-284.

McKernan R, McNeish J, Smith D. 2010. Pharma's developing interest in stem cells. Cell Stem Cell 6: 517-520.

Mohsin S, Siddiqi S, Collins B, Sussman MA. 2011. Empowering adult stem cells for myocardial regeneration. Circ Res 109: 1415-1428.

Ni TT, Rellinger EJ, Mukherjee A, Xie S, Stephens L, Thorne CA, Kim K, Hu J, Lee E, Marnett L, et al. 2011. Discovering small molecules that promote cardiomyocyte generation by modulating Wnt signaling. Chem Biol 18: $1658-1668$.

NIH. 2012. Stem/progenitor cell-derived human micro-organs and -tissues. RFA-RM-12-001, National Institutes of Health, Bethesda, MD.

Oh Y, Wei H, Ma D, Sun X, Liew R. 2012. Clinical applications of patient-specific induced pluripotent stem cells in cardiovascular medicine. Heart 98: 443-449.

Oikonomopoulos A, Sereti K-I, Conyers F, Bauer M, Liao A, Guan J, Crapps D, Han J-K, Dong H, Bayomy AF, et al. 2011. Wnt signaling exerts an antiproliferative effect on adult cardiac progenitor cells through IGFBP3. Circ Res 109: $1363-1374$.

Pammolli F, Magazzini L, Riccaboni M. 2011. The productivity crisis in pharmaceutical R\&D. Nat Rev Drug Discov 10: $428-438$.

Penn MS, Dong F, Klein S, Mayorga ME. 2011. Stem cells for myocardial regeneration. Clin Pharmacol Ther 90: 499501.

Penn MS, Mendelsohn FO, Schaer GL, Sherman W, Farr M, Pastore JM, Aras R, Rouy D, Clemens R, Cotts W. 2012. Re-establishment of SDF-1 expression through non-viral gene therapy improves clinical parameters through 12 months in patients with ischemic class III heart failure. J Cardiac Failure 18: S59-S60.

Porrello ER, Mahmoud AI, Simpson E, Hill JA, Richardson JA, Olson EN, Sadek HA. 2011. Transient regenerative potential of the neonatal mouse heart. Science 331: $1078-1080$.

Ptaszek LM, Mansour M, Ruskin JN, Chien KR. 2012. Towards regenerative therapy for cardiac disease. Lancet 379: 933-942.

Robinton DA, Daley GQ. 2012. The promise of induced pluripotent stem cells in research and therapy. Nature 481: 295-305.

Roobrouck VD, Ulloa-Montoya F, Verfaillie CM. 2008. Selfrenewal and differentiation capacity of young and aged stem cells. Exp Cell Res 314: 1937-1944.

Scannell JW, Blanckley A, Boldon H, Warrington B. 2012. Diagnosing the decline in pharmaceutical R\&D efficiency. Nat Rev Drug Discov 11: 191-200.

Schulz TC, Young HY, Agulnick AD, Babin MJ, Baetge EE, Bang AG, Bhoumik A, Cepa I, Cesario RM, Haakmeester C, et al. 2012. A scalable system for production of functional pancreatic progenitors from human embryonic stem cells. PloS ONE 7: e37004. 
Sensebé L, Krampera M, Schrezenmeier H, Bourin P, Giordano R. 2010. Mesenchymal stem cells for clinical application. Vox Sanguinis 98: 93-107.

Sewing A, Winchester T, Carnell P, Hampton D, Keighley W. 2008. Helping science to succeed: Improving processes in R\&D. Drug Discov Today 13: 227-233.

Smart N, Bollini S, Dubé KN, Vieira JM, Zhou B, Davidson S, Yellon D, Riegler J, Price AN, Lythgoe MF, et al. 2011. De novo cardiomyocytes from within the activated adult heart after injury. Nature 474: 640-644.

Smart N, Dubé KN, Riley PR. 2012. Epicardial progenitor cells in cardiac regeneration and neovascularisation. Vascul Pharmacol 58: 164-173.

Swinney DC, Anthony J. 2011. How were new medicines discovered? Nat Rev Drug Discov 10: 507-519.

Takahashi K, Yamanaka S. 2006. Induction of pluripotent stem cells from mouse embryonic and adult fibroblast cultures by defined factors. Cell 126: 663-676.

Taylor WF. 2011. The principles of scientific management. Dover, New York.

Thomson JA, Itskovitz-Eldor J, Shapiro SS, Waknitz MA, Swiergiel JJ, Marshall VS, Jones JM. 1998. Embryonic stem cell lines derived from human blastocysts. Science 282: $1145-1147$.

Tozer D. 2010. The challenges of product development and commercialization in a convergence technology world: Focus on regenerative medicine. Drug Discov Today 15: $587-589$.

U.S. Department of Health and Human Services, Food and Drug Administration. 2004. Innovation or stagnation:
Innovation at the Academia-Pharma Interface

Challenge and opportunity on the critical path to new medical products. U.S. Food and Drug Administration, Silver Spring, MD.

Vitale AM, Wolvetang E, Mackay-Sim A. 2011. Induced pluripotent stem cells: A new technology to study human diseases. Int J Biochem Cell Biol 43: 843-846.

von Gise A, Pu WT. 2012. Endocardial and epicardial epithelial to mesenchymal transitions in heart development and disease. Circ Res 110: 1628-1645.

Wei C, El Hindi S, Li J, Fornoni A, Goes N, Sageshima J, Maiguel D, Karumanchi SA, Yap H-K, Saleem M, et al. 2011. Circulating urokinase receptor as a cause of focal segmental glomerulosclerosis. Nat Med 17: 952-960.

Werner M, Ruffin M, West E. 2011. Regenerative medicine: A paradigm shift in healthcare. Drug Discovery World 13: $33-38$.

Willems E, Cabral-Teixeira J, Schade D, Cai W, Reeves P, Bushway PJ, Lanier M, Walsh C, Kirchhausen T, Izpisua Belmonte JC, et al. 2012. Small molecule-mediated TGF$\beta$ type II receptor degradation promotes cardiomyogenesis in embryonic stem cells. Cell Stem Cell 11: 242-252.

Wobus AM, Löser P. 2011. Present state and future perspectives of using pluripotent stem cells in toxicology research. Arch Toxicol 85: 79-117.

Yaniz-Galende E, Chen J, Chemaly ER, Liang L, Hulot JS, McCollum L, Arias T, Fuster V, Zsebo K, Hajjar RJ. 2012. Stem cell factor gene transfer promotes cardiac repair after myocardial infarction via in situ recruitment and expansion of c-kit ${ }^{+}$cells. Circ Res 111: 1434-1445. 


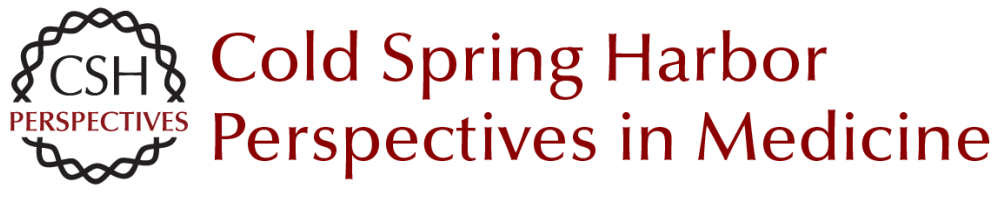

\section{Regenerative Medicine: Transforming the Drug Discovery and Development Paradigm}

Sotirios K. Karathanasis

Cold Spring Harb Perspect Med 2014; doi: 10.1101/cshperspect.a014084

Subject Collection The Biology of Heart Disease

The Genetic Basis of Aortic Aneurysm

Mark E. Lindsay and Harry C. Dietz

Personalized Genomes and Cardiovascular Disease

Kiran Musunuru

Complex Genetics and the Etiology of Human Congenital Heart Disease

Bruce D. Gelb and Wendy K. Chung

Genetic Networks Governing Heart Development Ashley J. Waardenberg, Mirana Ramialison, Romaric Bouveret, et al.

Heart Fields and Cardiac Morphogenesis Robert G. Kelly, Margaret E. Buckingham and Antoon F. Moorman

Regenerative Medicine: Transforming the Drug Discovery and Development Paradigm Sotirios K. Karathanasis

Myocardial Tissue Engineering: In Vitro Models Gordana Vunjak Novakovic, Thomas Eschenhagen and Christine Mummery

Pluripotent Stem Cell Models of Human Heart

Disease

Alessandra Moretti, Karl-Ludwig Laugwitz, Tatjana Dorn, et al.
Cardiac Cell Lineages that Form the Heart Sigolène M. Meilhac, Fabienne Lescroart, Cédric Blanpain, et al.

Synthetic Chemically Modified mRNA (modRNA):

Toward a New Technology Platform for

Cardiovascular Biology and Medicine

Kenneth R. Chien, Lior Zangi and Kathy O. Lui

Next-Generation Models of Human Cardiogenesis via Genome Editing Xiaojun Lian, Jiejia Xu, Jinsong Li, et al.

How to Make a Heart Valve: From Embryonic Development to Bioengineering of Living Valve

Substitutes

Donal MacGrogan, Guillermo Luxán, Anita Driessen-Mol, et al.

Insights into the Genetic Structure of Congenital Heart Disease from Human and Murine Studies on Monogenic Disorders

Terence Prendiville, Patrick Y. Jay and William T. $\mathrm{Pu}$

Cardiovascular Drug Discovery: A Perspective from a Research-Based Pharmaceutical Company G. Gromo, J. Mann and J.D. Fitzgerald

Genetics and Disease of Ventricular Muscle Diane Fatkin, Christine E. Seidman and Jonathan G. Seidman

Embryonic Heart Progenitors and Cardiogenesis Thomas Brade, Luna S. Pane, Alessandra Moretti, et al.

For additional articles in this collection, see http://perspectivesinmedicine.cshlp.org/cgi/collection/ 